\title{
Current status of FRIDA, diffraction limited NIR Instrument for the GTC
}

\author{
Beatriz Sánchez ${ }^{* a}$, José A. Acosta ${ }^{\mathrm{d}}$, Luis Carlos Álvarez ${ }^{\mathrm{a}}$, Vicente Bringas ${ }^{\mathrm{c}}$, Nicolás Cardiele \\ Adi Corrales ${ }^{c}$, Salvador Cuevas ${ }^{a}$, Oscar Chapa ${ }^{a}$, José Javier Díaz ${ }^{\mathrm{d}}$, Stephen S. Eikenberry ${ }^{\mathrm{e}}$, \\ Carmen Eliche ${ }^{\mathrm{f}}$, Carlos Espejo ${ }^{\mathrm{a}}$, Rubén Flores ${ }^{\mathrm{a}}$, Francisco Garzón ${ }^{\mathrm{d}}$, Peter Hammersley ${ }^{\mathrm{d}}$, \\ Carolina Keiman ${ }^{\mathrm{a}}$, Gerardo Lara ${ }^{\mathrm{a}}$, José Alberto López ${ }^{\mathrm{b}}$, Pablo López ${ }^{\mathrm{d}}$, Diana Lucero ${ }^{\mathrm{c}}$, \\ José Manuel Montoya $^{\mathrm{c}}$, Heidy Moreno ${ }^{\mathrm{d}}$, Sergio Pascual ${ }^{\mathrm{f}}$, Jesús Patrón ${ }^{\mathrm{d}}$, \\ Almudena Prieto ${ }^{\mathrm{d}}$, N. Raines ${ }^{\mathrm{e}}$, Alberto Rodríguez ${ }^{\mathrm{c}}$, Jorge Uribe ${ }^{\mathrm{c}}$, Alan Watson ${ }^{\mathrm{a}}$. \\ a Instituto de Astronomía, Universidad Nacional Autónoma de México, Apdo.Postal 70-264, 04510, \\ México D.F., México. \\ ${ }^{\mathrm{b}}$ Instituto de Astronomía, UNAM, Campus Ensenada. \\ ${ }^{c}$ Centro de Ingeniería y Desarrollo Industrial. Av. Playa Pie de la Cuesta 702, 76130, Qro. México. \\ ${ }^{\mathrm{d} I n s t i t u t o}$ de Astrofísica de Canarias, c/Vía Láctea s/n, E-38200 La Laguna, Tenerife, Spain \\ eAstronomy Department, University of Florida, 211 Bryant Space Center, PO Box 112055, \\ Gainesville FL, 32611-2055, USA \\ ${ }^{\mathrm{f}}$ Universidad Complutense de Madrid.
}

\begin{abstract}
FRIDA (inFRared Imager and Dissector for the Adaptive optics system of the Gran Telescopio Canarias) is designed as a diffraction limited instrument that will offer broad and narrow band imaging and integral field spectroscopy capabilities with low $(\mathrm{R} \sim 1,500)$, intermediate $(\mathrm{R} \sim 4,500)$ and high $(\mathrm{R} \sim 30,000)$ spectral resolutions to operate in the wavelength range $0.9-2.5 \mu \mathrm{m}$. The integral field unit is based on a monolithic image slicer. The imaging and IFS observing modes will use the same Teledyne $2 \mathrm{~K} \times 2 \mathrm{~K}$ detector. FRIDA will be based at the Nasmyth B platform of GTC, behind the AO system. The key scientific objectives of the instrument include studies of solar system bodies, low mass objects, circumstellar outflow phenomena in advanced stages of stellar evolution, active galactic nuclei, high redshift galaxies, resolved stellar populations, semi-detached binary systems, young stellar objects and star forming environments. FRIDA is a collaborative project between the main GTC partners, namely, Spain, México and Florida. In this paper, we present the status of the instrument design as it is currently being prepared for its manufacture, after an intensive prototypes' phase and design optimization. The CDR was held in September 2011.
\end{abstract}

Keywords: Infrared Instrumentation - Near Infrared; Imaging; Integral Field Spectroscopy; IFU; GTC; Diffraction Limited

\section{INTRODUCTION}

The GTC is the single largest operating optical/infrared telescope in the world and as such it has two distinct advantages in the study of the local and distant universe. The first is that the light grasp of a telescope scales as the square of the diameter, and so the GTC will gather more photons than any other optical/infrared telescope. The second is that the diffraction limit of a telescope scales as the inverse of the diameter and so, once the adaptive optics system is operating, the GTC will reach the finest resolution of any infrared telescope. Thus, the GTC will be ideally suited for high spatial resolution studies of both faint and bright objects.

*beatriz@astro.unam.mx; phone +52 55562239 10; fax +52 5556160653

Ground-based and Airborne Instrumentation for Astronomy IV, edited by lan S. McLean, Suzanne K. Ramsay, Hideki Takami, Proc. of SPIE Vol. 8446, 844624 - (C) 2012 SPIE · CCC code: 0277-786X/12/\$18 · doi: 10.1117/12.926499 
The GTC project office is developing the GTCAO system ${ }^{1}$ that will be essential for GTC to reach its full potential. At first the GTCAO system will be a single-conjugate system operating with an optical wave front sensor and natural guide stars. This system is expected to provide a partially-corrected beam with an on-axis Strehl ratio in $\mathrm{K}$ of 0.65 with a bright guide star and 0.45 with a guide star of $\mathrm{mR}=15$. As the system will be a single-conjugate system, the corrected field is not expected to be larger than about 20 arcsec in radius.

FRIDA was conceived as the first instrument ${ }^{2}$ for the initial adaptive optics system of the GTC. It was agreed that the partner institutions in GTC, namely: IA-UNAM, IAC, UF as well as UCM jointly build the instrument. During the initial phase of the project LAOMP also participated. FRIDA inherits much of the existing expertise in instrumentation development of these institutions for GTC.

\section{CHARACTERISTICS}

FRIDA will be a common-user near infrared imager and integral field spectrograph and will cover the wavelength range from 0.9 to 2.5 microns. It has identified imaging and integral field spectroscopy as the most interesting modes from the perspective of science; these primary modes will drive the design of the instrument. FRIDA will be installed at the Nasmyth B platform behind the GTCAO system. It will use diffraction-limited optics to avoid degrading the high Strehl ratios delivered by the GTCAO system in the near infrared; with these characteristics we anticipate that FRIDA will be a highly competitive instrument.

FRIDA will provide a direct imaging mode with broad-band and narrow-band filters, selectable spatial scales of 0.010 arcsec per pixel and 0.020 arcsec per pixel, and corresponding fields of view of $20.48 \times 20.48$ arcsec and $40.96 \times 40.96$ arcsec. The finer scale will provide adequate sampling of the almost diffraction-limited core in $\mathrm{J}$ and $\mathrm{H}$ bands and the medium scale will provide adequate sampling in K band. A third camera with a coarse scale of 0.040 arcsec per pixel will also be available. However, this camera has been designed mainly for acquisition purposes in the IFS mode and for cases when only partial AO correction is achieved due to either poor atmospheric or inadequate guide star conditions. This coarse camera will deliver good image quality in the restricted IFU field of view.

FRIDA will provide an integral field spectroscopy mode using an image slicer ${ }^{3}$. The selectable spatial scales parallel to the slices will be 0.010 arcsec per pixel, 0.020 arcsec per pixel and 0.040 arcsec per pixel and the corresponding spatial scales perpendicular to the slices will be 0.020 arcsec per slice, 0.040 arcsec per slice and 0.080 arcsec per slice. Each slice will project to two pixels in the spectral direction. For an image slicer with thirty slices, these scales yield fields of view of $0.65 \times 0.60 \mathrm{arcsec}, 1.30 \times 1.20 \mathrm{arcsec}$ and $2.60 \times 2.40 \mathrm{arcsec}$. The available spectral resolutions will be $\mathrm{R} \sim 1500$ to cover the $\mathrm{H}+\mathrm{K}$ bands, $\mathrm{R} \sim 4500$ over each of the $\mathrm{Z}, \mathrm{J}, \mathrm{H} \& \mathrm{~K}$ bands and $\mathrm{R} \sim 30,000$ over selectable windows from 1.4 to 2.5 microns. The combination of high spectral resolution and high spatial resolution will be a unique capability of FRIDA.

\section{SCIENCE CASES}

FRIDA will reveal astrophysical phenomena on angular scales as small as 0.010 arcsec. The operating wavelength range of FRIDA will expose dusty environments and will probe a spectral range rich in stellar absorption lines and in emission lines from many different species and gas phases. With these characteristics, FRIDA will be able to tackle a large number of key astrophysical problems.

The interest for FRIDA in the GTC community is broad. The FRIDA scientific case ranges from solar system bodies to high redshift systems, including close binary systems, young stellar objects, circunstellar phenomena in advanced stages of stellar evolution, resolved stellar populations and active galactic nuclei.

\section{ARCHITECTURE AND DESIGN OVERVIEW}

The GTCAO corrected beam is directed to the entrance window. The optical components and mechanisms will be mounted inside the cryostat and on the optical bench, only the wavelength calibration unit will be outside the cryostat. 
The FRIDA optical concept ${ }^{4}$ consists of a classical refractive Collimator-Camera (Medium Camera) that images the GTC pupil in the pre-optics pupil plane and the GTCAO output image in the input object plane of the spectrograph. The preoptics has four cameras to provide the requested spatial scales and perform a linear amplification. These are the Coarse, Medium, Fine and Pupil Cameras. The optics for the Collimator-Medium Camera is fixed to the optical bench, the others cameras are mounted on a camera wheel that inserts/removes them on the optical beam. The spectrograph is a double pass system performing the collimator and the camera.

The switching mode mechanism (see Figure 4.1) sends the light to the detector in the imaging mode, for the IFS mode the light passes through the second focal plane to reach the IFU. The FRIDA IFU divides the IFS field into 30 slices and creates the output pseudo-slit of the spectrograph. The spectrograph grating carrousel will hold 2 high resolution gratings, 4 medium resolution gratings, 1 low resolution grating and 2 IFU mapping mirrors. The dispersed light comes back from double pass spectrograph and the switching mechanism finally sends the beam on the Hawaii-2 detector array. This detector is mounted on a focus mechanism.

All refractive components collimator, cameras and spectrograph are based on airspace doublets using two cryogenic matched materials: CaF2 and Ohara S-FTM16.

The optical scheme for FRIDA is shown in Figure 4.1 for the Imaging Mode and for the IFS Mode, both in the Medium Camera Configuration, both modes using a single detector, Teledyne $\mathrm{HgCdTe}$.
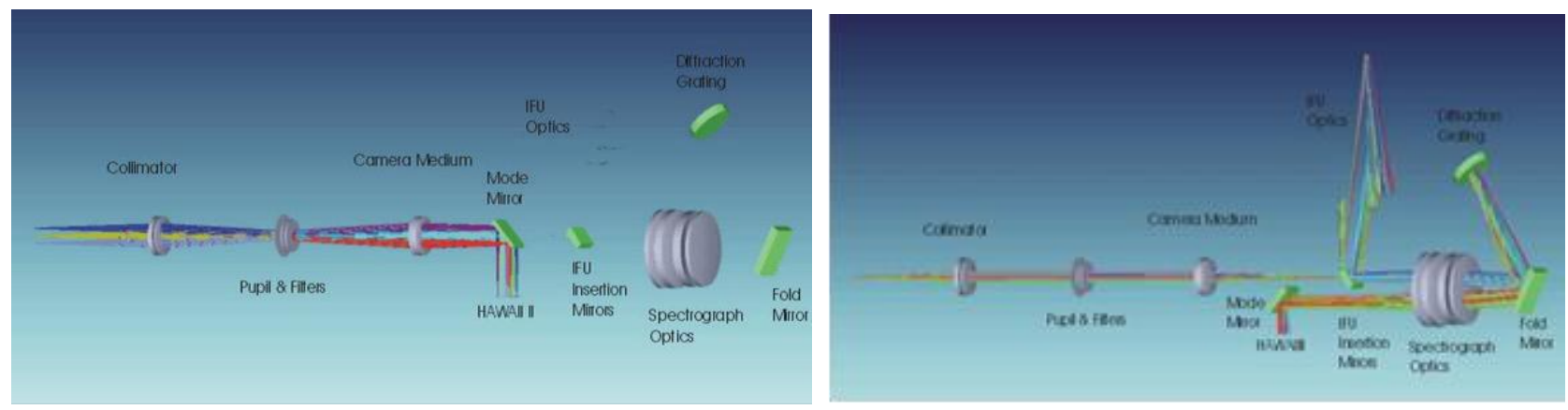

Figure 4.1 Optical Layout, Imaging Mode (left) and IFS Mode (right) both in Medium Configuration.

The instrument will be attached to the Nasmyth B platform through a support structure with alignment interfaces that will ensure the optical alignment with the GTCAO. Figure 4.2 shows the isometric view of the cryostat and its support structure on the platform. The main body of the cryostat has a frontal cylindrical section that is a dewar extension, this extension allows the corrected GTCAO beam to reach the right focal plane position. The dewar main body is closed with upper and lower covers. Attached to the dewar upper cover is the turbo molecular pump and two feed-through connector plates are in the lower cover. In the dewar main body, there are two cryocoolers located in the opposite sides of the lateral walls. In addition, the bellows for LN2 filling, pressure gauges, valves and the detector connector plate are located in the lateral walls.

The FRIDA cold structure is mounted inside a vacuum dewar. Three support trusses allow attaching the optical bench to the cryostat lower cover and maintain the thermal insulation. The LN2 container is below the optical bench for efficient initial cooling, whereas the two cryocooler cold heads will be used to maintain the FRIDA components at the working temperature.

The instrument layout is based on a rectangular cryostat having a flat optical bench (see Figure 4.3). The optical beam enters parallel to the optical bench in a horizontal position. This configuration has the g-vector perpendicular to the optical bench and allows mounting and aligning the instrument subsystems in the same final position operation position.

The Figure 4.4 shows the cryostat dimensions. 


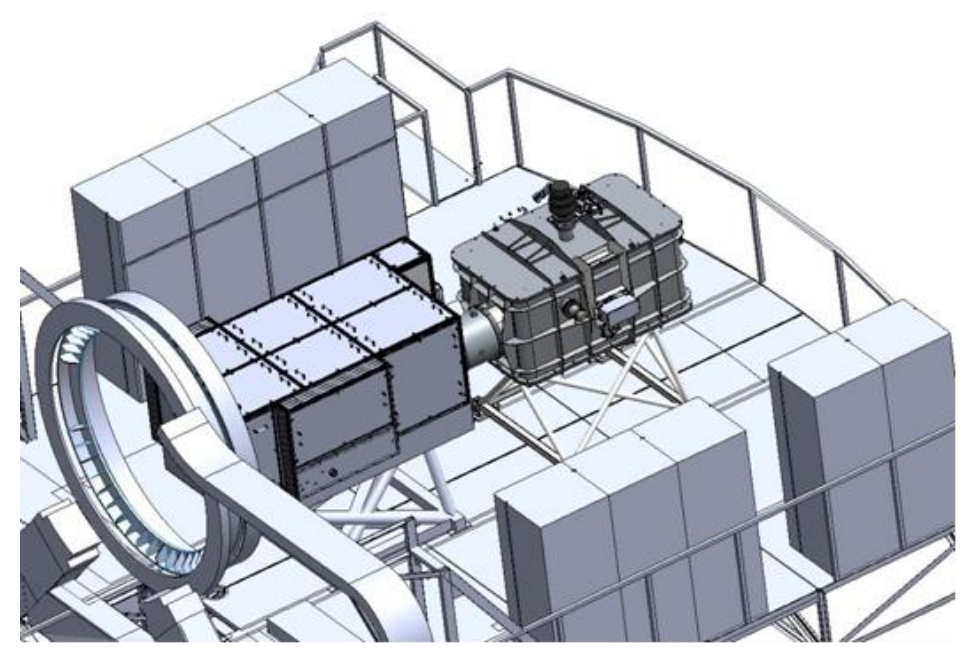

Figure 4.2 FRIDA at the Nasmyth platform B of the GTC, behind to GCTAO System.

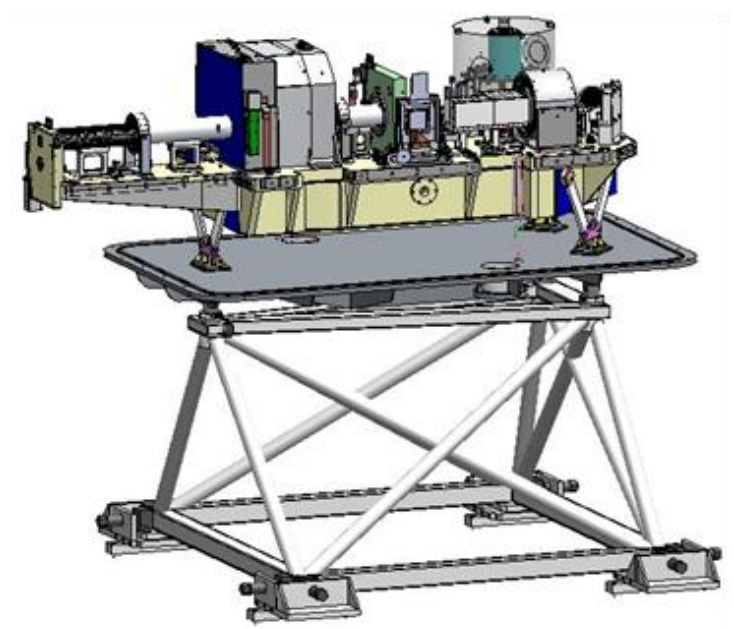

FRIDA 4.3 optical bench with all cold subsystems on the dewar's lower plate and the support structure.

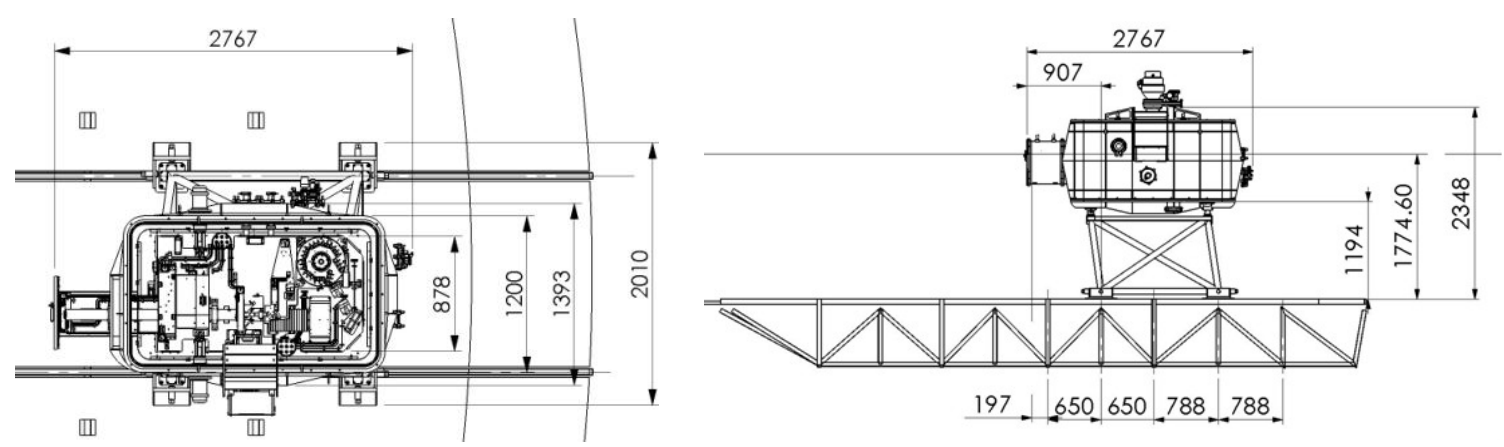

Figure 4.4 Cryostat exterior dimensions. Upper view, the dimensions are in millimeters (right). FRIDA high dimensions. Lateral view. Dimensions are in millimeters (right) 


\section{PROJECT ORGANIZATION ESQUEME}

The Universidad Nacional Autónoma de México (UNAM) is the scientific and technical leader institution for FRIDA and as such the UNAM has established formal agreements for the development of the corresponding work packages with the participating institutions: The Instituto de Astrofísico de Canarias (IAC, Spain), El Centro de Ingeniería y Desarrollo Industrial (CIDESI, México), The Universidad Complutense de Madrid (UCM, Spain) and The University of Florida (UF, USA). These agreements have been formalized and signed. The organization scheme of FRIDA requires a strict control of the responsibilities and clear definitions of the work package for each participating institution and their interfaces, deadlines and budget to ensure its successfully conclusion.

The purpose of the FRIDA team is to unite and capitalize the expertise and synergies of the participating institutions: The IAC is responsible for coordinating all the activities related to the development of the FRIDA control system, including those related to the Hawaii II detector system and the high level software for the control mechanisms and the sequencer, the inspector panel, the exposure time calculator, etc. The UF is responsible for the development of the FRIDA-IFU, including its fabrication, integration and final testing. UF also are in charge of the focus mechanism. CIDESI is responsible for the complete mechanical work package, design and manufacturing of all the mechanical elements (cryostat, mechanisms, camera barrels, etc.). UCM is developing the data factory pipeline. The IA-UNAM is in charge of the coordination and management of the FRIDA science and engineering teams, it is also responsible for the full optical design, procurement and manufacture of the optical elements, coordination of the assembly, integration and verification (AIV) tasks and participates in the control work packages with the IAC in the areas of mechanism control (low level software), instrument cabling and house-keeping electronics.

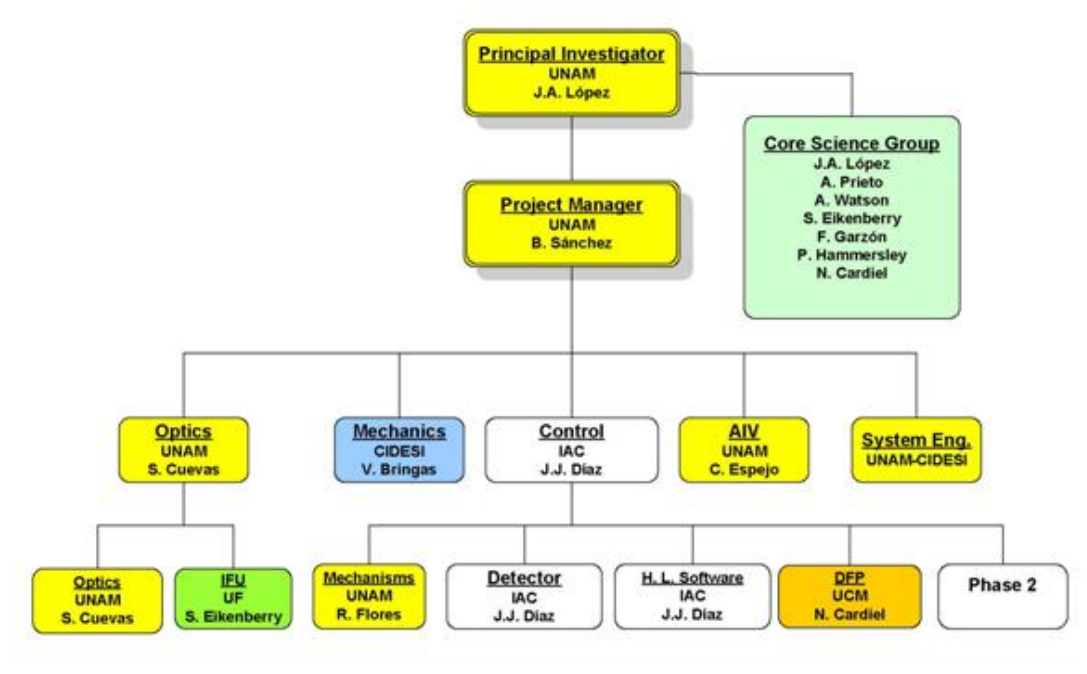

Figure 5.1 FRIDA Organization Chart

The project manager and principal investigator have maintained a close contact with each members of the FRIDA working team to keep an agile flux of project information through regular project meetings. With the same purpose a database with the internal project documentation has been constantly updated.

\section{INSTRUMENT CURRENT STATUS}

The FRIDA concept project was accepted officially in March 2005 by GRANTECAN. The conceptual optical design was reviewed on July 2006; several changes were suggested mainly for the spectrograph design and the IFU design. The Preliminary Design Review (PDR) was in August 2007, this review led to changes in the design of the support structure and the optical and mechanical design of the cameras wheel and barrels. 
The preliminary design review also led to the development of prototypes for some key systems such as the focal plane wheel and the gratings carousel mechanism that are the subsystems with the tighter specification. Since all the refracting FRIDA optical subsystem are based in doublets formed by one CaF2 lens and other S-FTM16 lens, it was decided to include also a prototype of the collimator optomechanics ${ }^{5}$ as well as the focus mechanism. All the prototype design concepts have been successfully transferred to the subsystems final designs (see Figures 5.2 and 5.3).

IA-UNAM did not have adequate cryogenics facilities for testing the prototypes and the FRIDA subsystems, therefore it was necessary to design and build two cryostats (Colibrí and FCTF) to be used for components and subsystems verification. These cryostats were designed and built at IA-UNAM and CIDESI and have been used successfully for its intended purpose. The experience acquired in designing and manufacturing these cryostats will be fully applied to the development of the final FRIDA cryostat and its cryogenic subsystems.
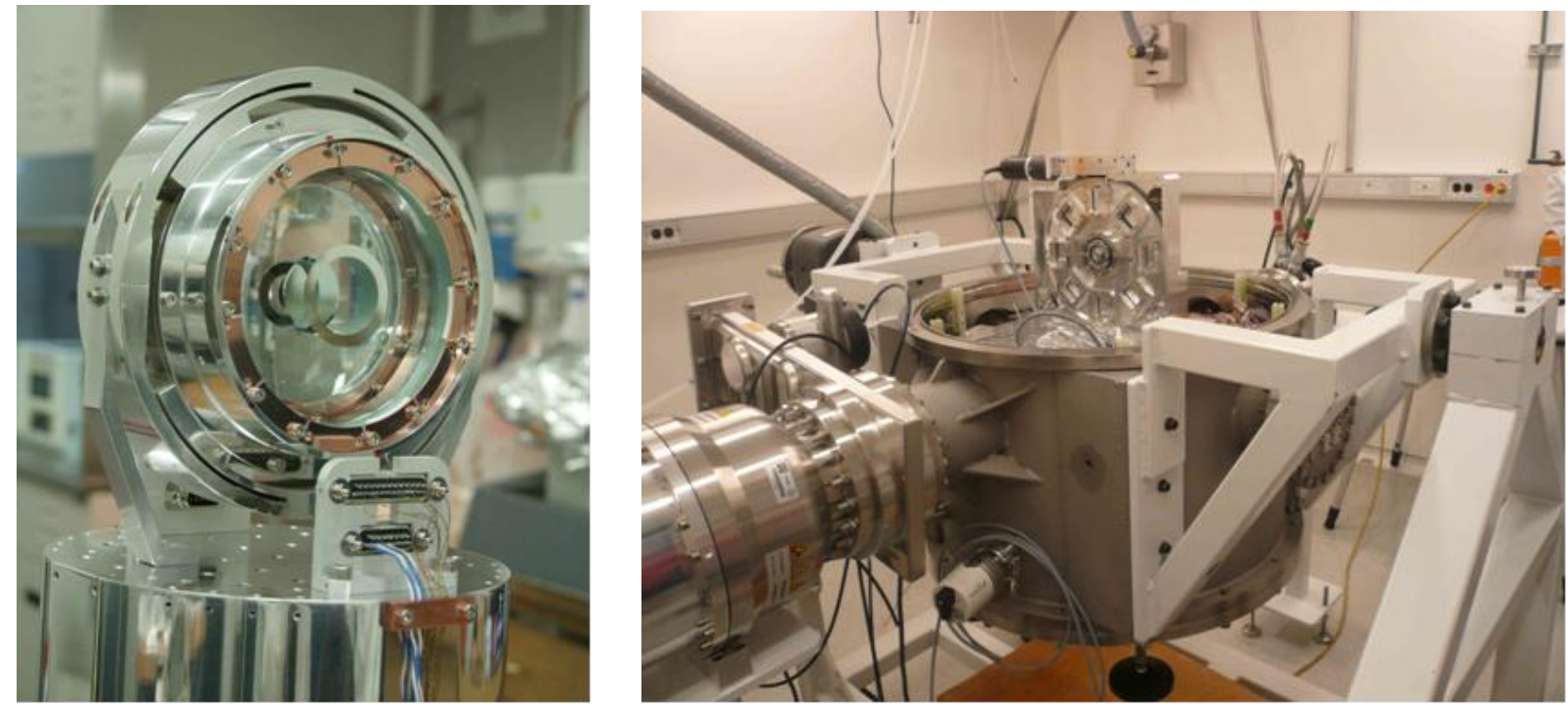

Figure 6.1 Left, collimator Prototype on Colibrí's cold bench. Right, focal plane wheel on FCTF cold bench
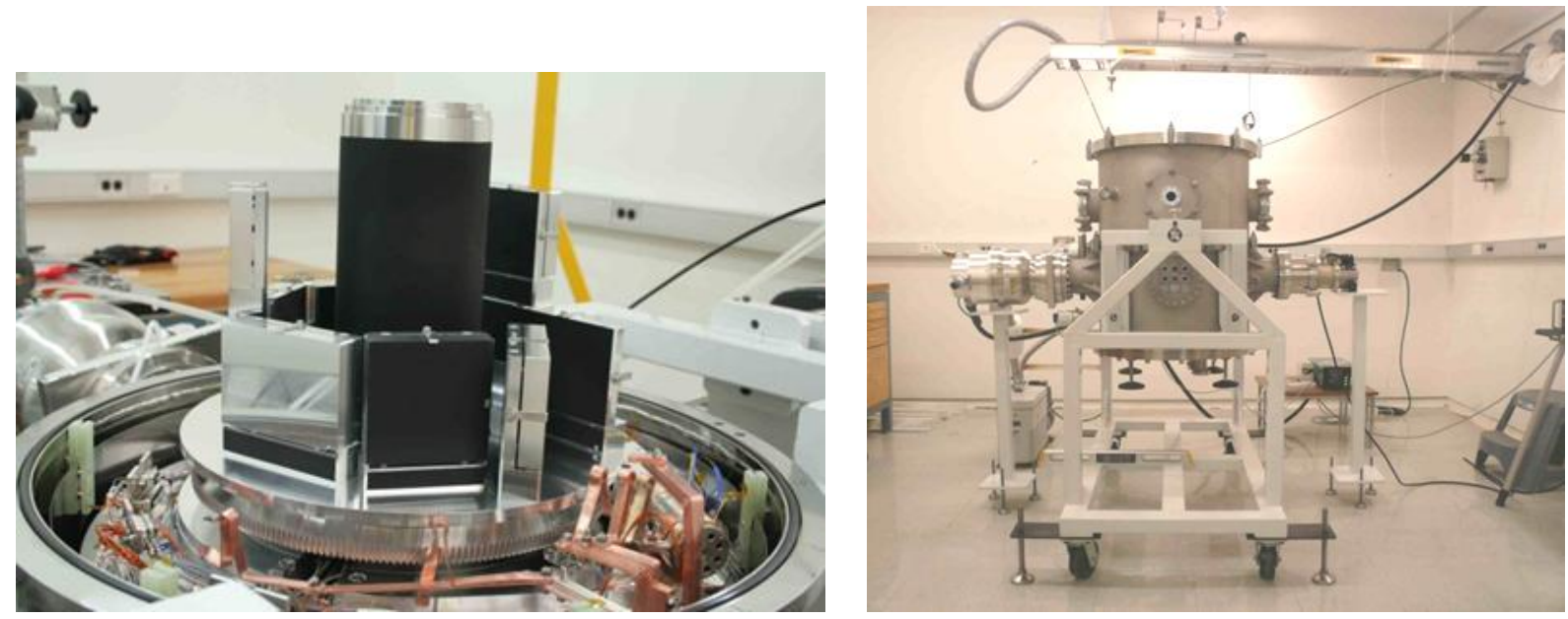

Figure 6.2 Grating Carrousel on FCTF's clod bench and the FCTF cryostat. 
The critical design review was held in México City last September with positive results. The FRIDA team has been working on the review panel recommendations. We are at present aiming to close all the pending tasks from the CDR and have begun the stage of procurement and fabrication of subsystems.

In order to minimize risks, delays and to have more control on procurement and manufacture processes, the instrument will be developed within FRIDA's institutions. Electronics control and software will be developed by IAC, UCM and IAUNAM. All lenses will be manufactured by UNAM or under UNAM's supervision in México. Only the IFU manufacture will be subcontracted by UF under supervision of S.S. Eikenberry and S. Cuevas. CIDESI has the required resources and infrastructure to carry out the mechanical manufacture of the whole instrument.

The integration and tests at subsystem level are scheduled by 2013 and to system level are scheduled by 2014 . The laboratory acceptance is scheduled by December 2014 and the GTC site acceptance and commissioning will be in 2015.

\section{ACKNOWLEDGEMENTS}

We are grateful to Grupo Santander (Spain) through Encuentros Astrofísicos Blas Cabrera (UNAM-IAC) and our home institutions for their support. We also gratefully acknowledge partial funding for this project through generous grants from PAPITT-UNAM (grant IT116311) and CONACYT grant INFR-2009-01-122664)

\section{REFERENCES}

[1] Devaney, N., Bello, D., Femenias, B., et al., "Preliminary design and plans for the GTC Adaptive Optics System", Proc. SPIE 5490, 913 (2004).

[2] López, J.A., Bringas, V., Cuevas, S., et al., "FRIDA: Integral-field and spectrograph and imager for the adaptive optics system Gran Telescopio Canarias", Proc. SPIE 6269, 119L (2007).

[3] Cuevas S., Eikenberry, S.S., et al "FRIDA Integral Field Unit Opto-mechanical Design" Proc. SPIE 8450-67, (2012).

[4] Cuevas S., Eikenberry, S.S., Sánchez, B., et al., "Optical design of FRIDA, the integral-field spectrograph and imager for the AO system of the Gran Telescopio Canarias", Proc. SPIE 7014, 70146D (2008).

[5] Cuevas S., Álvarez, L.C., et al., "Cryogenic Tests on FRIDA building blocks" Proc. SPIE 8450-67, (2012).

[6] Flores-Meza, R., Cuevas, S., et al., "Electronics and mechanisms control system for FRIDA (inFRared Imager and Dissector for Adaptive optics)". Proc. SPIE 8451-138, (2012). 